\title{
Role of $\alpha$-crystallin $B$ as a regulatory switch in modulating cardiomyocyte apoptosis by mitochondria or endoplasmic reticulum during cardiac hypertrophy and myocardial infarction
}

\author{
A Mitra ${ }^{1}$, T Basak ${ }^{2}$, K Datta ${ }^{1}$, S Naskar ${ }^{1}$, S Sengupta ${ }^{2}$ and S Sarkar ${ }^{\star, 1}$
}

Cardiac hypertrophy and myocardial infarction (MI) are two major causes of heart failure with different etiologies. However, the molecular mechanisms associated with these two diseases are not yet fully understood. So, this study was designed to decipher the process of cardiomyocyte apoptosis during cardiac hypertrophy and MI in vivo. Our study revealed that mitochondrial outer membrane channel protein voltage-dependent anion channel-1 (VDAC1) was upregulated exclusively during cardiac hypertrophy, whereas $78 \mathrm{kDa}$ glucose-regulated protein (GRP78) was exclusively upregulated during Ml, which is an important upstream regulator of the endoplasmic reticulum (ER) stress pathway. Further downstream analysis revealed that mitochondrial pathway of apoptosis is instrumental in case of hypertrophy, whereas ER stress-induced apoptosis is predominant during Ml, which was confirmed by treatment with either siRNA against VDAC1 or ER stress inhibitor tauroursodeoxycholic acid (TUDCA). Very interestingly, our data also showed that the expression and interaction of small heat-shock protein $\alpha$-crystallin B (CRYAB) with VDAC1 was much more pronounced during MI compared with either hypertrophy or control. The study demonstrated for the first time that two different organelles-mitochondria and ER have predominant roles in mediating cardiomyocyte death signaling during hypertrophy and Ml, respectively, and activation of CRYAB acts as a molecular switch in bypassing mitochondrial pathway of apoptosis during MI.

Cell Death and Disease (2013) 4, e582; doi:10.1038/cddis.2013.114; published online 4 April 2013

Subject Category: Experimental Medicine

Heart diseases leading to heart failure remain the leading cause of death worldwide. Cardiac hypertrophy and myocardial infarction (MI) are the two major causes of heart failure with differential genetic etiologies. MI reflects a loss in cardiac myocyte population caused by prolonged ischemia due to an imbalance between oxygenated blood supply and demand, ${ }^{1}$ whereas cardiac hypertrophy is an adaptive response of the heart muscle to a wide variety of intrinsic and extrinsic stimuli. Although hypertrophy of the heart muscle is initially beneficial during early growth, prolonged hypertrophy is potentially deleterious and leads to heart failure. ${ }^{2}$

It has been reported that the apoptotic machinery is turned on during transition of cardiac hypertrophy to heart failure. ${ }^{3}$ Several investigators have proposed various pathways of cardiomyocyte apoptosis during transition of hypertrophy to heart failure by the activation of sodium/hydrogen exchanger- $1,{ }^{4}$ upregulation of apoptosis-inducing factor $^{5}$ and
Bcl-2-associated X-protein (Bax), ${ }^{6}$ dysfunction in the electron transport chain ${ }^{7}$ and activation of protein kinase $\mathrm{C}-\delta .{ }^{8}$ Involvement of p53 and its interaction with nuclear factor $\kappa$-light-chain enhancer of activated B cells (NF- $\kappa \mathrm{B})$ by $\mathrm{p} 300$ was shown recently to be a major regulator of apoptosis in hypertrophic cardiomyocytes. ${ }^{3}$

Conversely, loss of cardiomyocytes during MI has been mainly attributed to necrosis, ${ }^{9}$ although involvement of reactive oxygen species, ${ }^{10}$ P38-mitogen-activated protein kinase $(\mathrm{MAPK})^{11}$ and NF- $\kappa \mathrm{B}$ activation in cardiomyocyte apoptosis during $\mathrm{Ml}$ has been reported. ${ }^{12}$ Recently, it has been suggested that few small heat-shock proteins (sHSPs) have instrumental role in the regulation of apoptotic signaling in stressed cardiac myocytes, which include $\alpha$-crystallin $B$ (CRYAB). ${ }^{13}$ CRYAB has been shown to perform protective function in cardiomyocytes by maintaining sarcomeric elasticity, mitochondrial integrity and also homeostasize redox imbalance by binding to different target molecules. ${ }^{14-16}$

\footnotetext{
${ }^{1}$ Department of Zoology, University of Calcutta, 35, B.C. Road, Kolkata 700 019, India and ${ }^{2}$ Genomics and Molecular Medicine unit, CSIR-IGIB, Sukhdev Vihar, Mathura Road, New Delhi 110 020, India

*Corresponding author: S Sarkar, Department of Zoology, University of Calcutta, 35, Ballygunge Circular Road, Kolkata 700019, West Bengal, India. Tel: +91 332475 3681; Fax: +9133 2476 4419; E-mail: sagartirtha.sarkar@ gmail.com

Keywords: cardiomyocyte apoptosis; cardiac hypertrophy; myocardial infarction; $\alpha$-crystallin B

Abbreviations: MI, myocardial infarction; VDAC1, voltage-dependent anion channel-1; GRP78, $78 \mathrm{kDa}$ glucose-regulated protein; ER, endoplasmic reticulum; CRYAB, $\alpha$-crystallin B; Bax, Bcl-2-associated X-protein; NF- $\kappa$ B, nuclear factor $\kappa$-light-chain enhancer of activated B cells; MAPK, mitogen-activated protein kinase; sHSPs, small heat-shock proteins; TTC, 2,3,5-triphenyltetrazolium chloride; TUDCA, tauroursodeoxycholic acid; Angll, angiotensin II; ANF, atrial natriuretic factor; $\beta$ MHC, $\beta$-myosin heavy chain; PBS, phosphate-buffered saline; ATF6, activating transcription factor 6; PERK, ER-resident PKR-like elF2 $\alpha$ kinase; CHOP, CCAAT/ enhancer-binding protein homologous protein; JNK, c-Jun N-terminal kinase; XBP1, X-Box-binding protein 1; IRE1, inositol-requiring enzyme 1; ATF4, activating transcription factor 4; TRAF2, tumor necrosis factor receptor-associated factor 2; PARP, poly ADP ribose polymerase; RPL32, 60S ribosomal protein L32; ANT, adenine nucleotide translocator; LVDD, left ventricular diastolic dimension; \%FS, percentage fractional shortening

Received 03.11.12; revised 19.2.13; accepted 20.2.13; Edited by A Stephanou
} 
The present study was directed to identify the difference in cardiomyocyte apoptotic machinery in two etiologically different cardiac diseases (hypertrophy and MI). Furthermore, analysis of downstream signaling pathways revealed that CRYAB has a crucial role in regulating cardiomyocyte apoptosis during hypertrophy and MI. This is the first report identifying novel molecular mechanisms for cardiomyocyte apoptosis, with precise role of CRYAB as a chief regulator for differential modes of myocyte apoptosis in two cardiac disease forms.

\section{Results}

Assessment of MI and hypertrophy in vivo and in vitro. Significantly large areas of infarct were revealed by triphenyltetrazolium chloride (TTC) staining in the left ventricles of both isoproterenol-treated and LAD-ligated rats $(23 \pm 1.58 \%$ and $27 \pm 2.1 \%$ of total left ventricular area, respectively; Supplementary Figure S1A) along with a significantly increased expression of the ischemia marker xanthine oxidase during isoproterenol treatment $(3.62 \pm 0.07$-fold $), \quad L A D$ ligation (3.3 \pm 0.18 -fold) and hypoxia (3.1 \pm 0.05 -fold) compared with respective controls. Xanthine oxidase expression was unaltered during hypertrophy (Supplementary Figure S1B). Generation of hypertrophy on the other hand, was ascertained by significant increase in the heart weight to body weight ratio, increased cardiomyocyte crosssectional area and significant increase in the expression of hypertrophy marker genes atrial natriuretic factor (ANF) and $\beta$-myosin heavy chain $(\beta-M H C)$ compared with respective controls, both in vivo as well as in vitro as shown earlier. ${ }^{3}$ Compromised cardiac function during hypertrophy was ascertained by M-mode echocardiographic analysis with significant increase in left ventricular diastolic dimension (LVDD; $0.64 \pm 0.05 \mathrm{~cm} ; P<0.05$ ) compared with either control $(0.46 \pm 0.03 \mathrm{~cm})$ or $\mathrm{Ml}(0.51 \pm 0.06 \mathrm{~cm})$, and significant decrease in percentage fractional shortening (\%FS) during hypertrophy $(29.5 \pm 1.92 \% ; P<0.05)$ compared with either control $(63 \pm 4.22 \%)$ or $\mathrm{Ml}(48 \pm 3.79 \%)$.

Differential expression of VDAC1, GRP78 and CRYAB during hypertrophy and MI. On the basis of the leads obtained from a comparative proteome profiling between hypertrophy and MI performed by us (unpublished data), the levels of voltage-dependent anion channel-1 (VDAC1) and $78 \mathrm{kDa}$ glucose-regulated protein (GRP78) were estimated in our experimental animal groups by western blot analysis. In agreement with the proteomics data, VDAC1 expression showed significant increase (3.08 \pm 0.06 -fold) exclusively during hypertrophy, whereas significant upregulation of GRP78 expression was observed during $\mathrm{Ml}$ in isoproterenol-treated (3.4 \pm 0.08 -fold) or LAD-ligated rats $(3.72 \pm$ 0.17-fold) compared with either control or hypertrophy (Figures 1a and b). Furthermore, significant increase in expression of CRYAB was observed during both hypertrophy $(1.31 \pm 0.07$-fold) and $\mathrm{Ml}$ (isoproterenol treatment $-4.82 \pm$ 0.26 -fold; LAD ligation - 3.91 \pm 0.18 -fold) in tune with our proteomics data (Figures 1a and b).

Evidence of apoptosis during both hypertrophy and MI. Though mitochondrial VDAC1 and endoplasmic reticulum (ER) stress marker GRP78 were upregulated during a
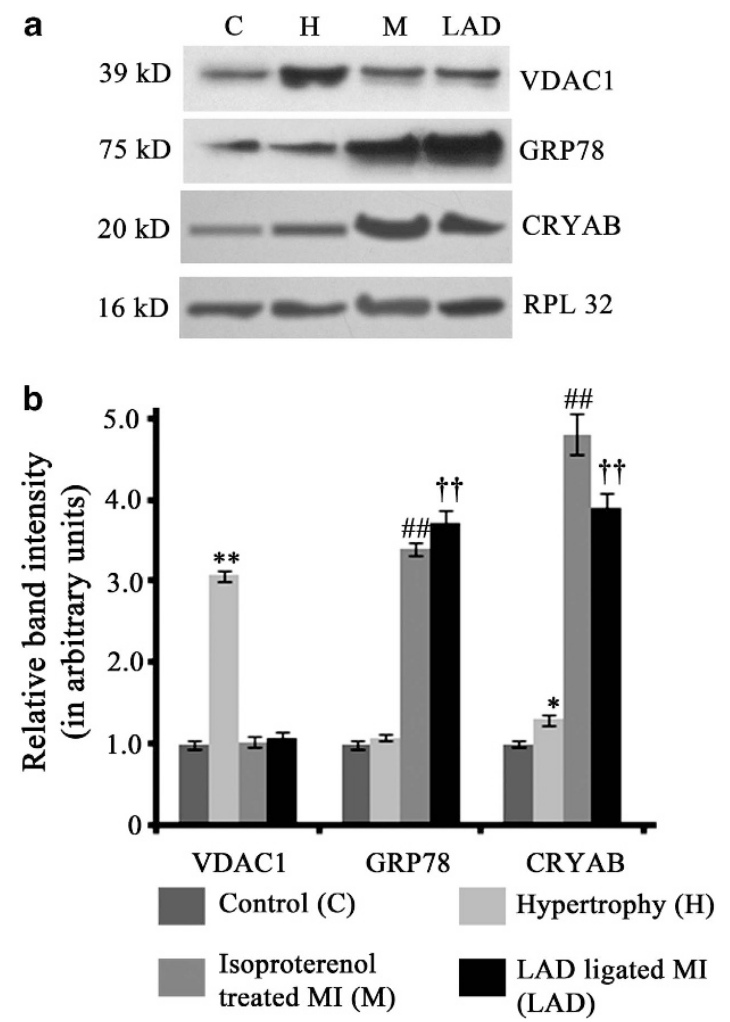

Figure 1 (a) Western blot analyses showing change in the expression of VDAC1, GRP78 and CRYAB in control $(C)$, hypertrophy $(H)$, isoproterenol-treated $\mathrm{MI}(\mathrm{M})$ and $\mathrm{LAD}$-ligated $\mathrm{MI}(\mathrm{LAD})$ in accordance with the proteomics data. (b) Graphs showing relative band intensity of (i) VDAC1, (ii) GRP78 and (iii) CRYAB, as revealed by western blot analyses. $\left({ }^{* *} P<0.01\right.$ with respect to $\mathrm{H}$ versus $\mathrm{C}, \mathrm{H}$ versus $M$ and $H$ versus $L A D$; \#\#P<0.01 with respect to $M$ versus $H$ and $M$ versus $C$; $\dagger \dagger P<0.01$ with respect to $L A D$ versus $H$ and $L A D$ versus $C$ )

hypertrophy and $\mathrm{MI}$, whether this upregulation resulted in dysfunction of mitochondria or ER leading to myocyte apoptosis was not clear. Therefore, some apoptotic proteins were analyzed by immunoblotting. As shown in Figure 2a, cleavage of caspase 3 (19 kDa fragment), a major regulator of apoptosis was observed during hypertrophy and MI. Similar trend was observed in caspase 3 activity assay, which showed increased activity during hypertrophy $(2.12 \pm 0.12$-fold), isoproterenol treatment (1.64 \pm 0.1 -fold) and LAD ligation (1.59 \pm 0.09 -fold) compared with control (Figure $2 b$ ). Poly ADP ribose polymerase (PARP), another apoptotic marker was also found to be cleaved during hypertrophy and MI. Similar results were observed in vitro in case of both angiotensin II (Angll)-treated and hypoxic cardiomyocytes (Figure 2a). Significant increase in the number of TUNEL-positive nuclei was also observed during hypertrophy $(37 \pm 2.58 \%)$ and $\mathrm{MI}(28 \pm 2.2 \%)$ compared with control samples ( $2 \pm 0.18 \%$; Figure $2 \mathrm{c})$.

Cardiomyocyte apoptosis during hypertrophy is mediated via VDAC1-influenced mitochondrial pathway. On the basis of the fact that myocyte apoptosis was evident during hypertrophy with exclusively upregulated expression of mitochondrial outer membrane channel VDAC1, the mitochondrial apoptotic pathway was assessed in the three 

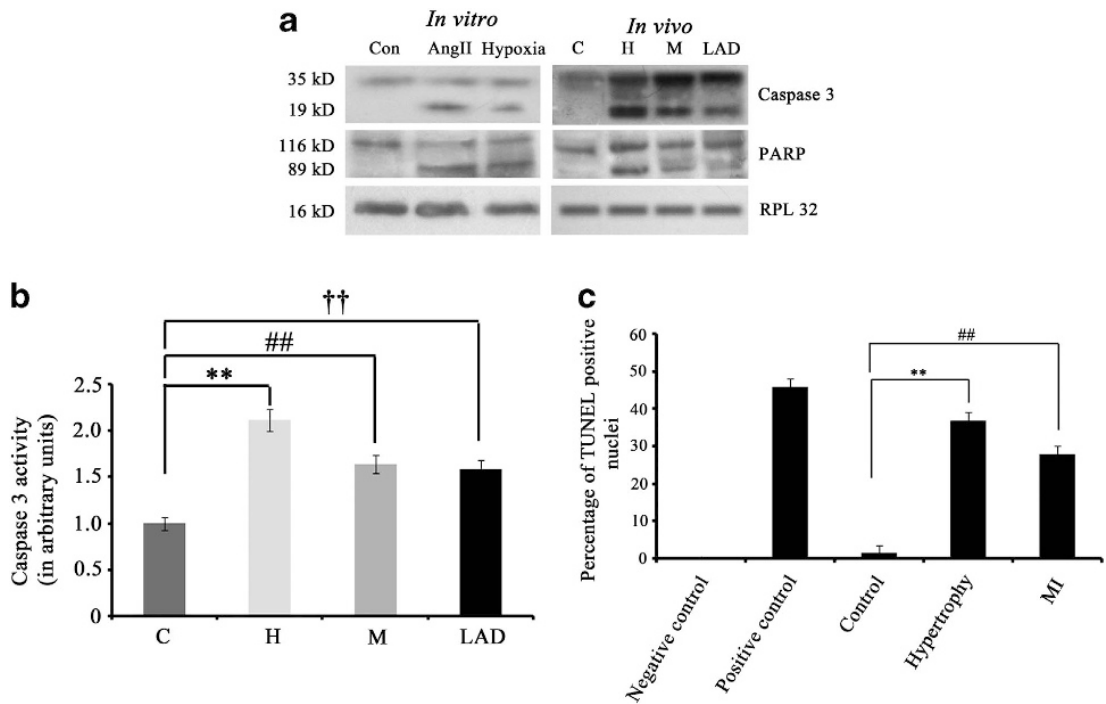

Figure 2 (a) Cardiomyocyte apoptosis occurs during hypertrophy and Ml. Western blot analyses showing cleavage of active caspase 3 (19 kDa) and PARP (89 kDa) proteins in both in vitro and in vivo hypertrophy and MI (isopreternol-treated and LAD) models. No such cleaved product was observed in control. RPL32 was used as internal loading control. Data is representative of three independent experiments. (b) Graph showing caspase 3 activity in control $(\mathrm{C})$, hypertrophy $(\mathrm{H})$, isoproterenol-treated $\mathrm{Ml}(\mathrm{M})$ and LAD-ligated MI (LAD). (c) Graph showing percentage of TUNEL-positive cells in paraffin-embedded tissue section of $\mathrm{C}, \mathrm{H}$ and $\mathrm{MI}$. (* $P<0.01$ with respect to $\mathrm{H}$ versus $\mathrm{C}$; $\# \# P<0.01$ with respect to $M$ versus $C ; \dagger \dagger P<0.01$ with respect to $L A D$ versus $C$ )

groups. Immunoblot analyses showed significantly increased expression of Bax ( $4 \pm 0.09$-fold) and increased cytosolic/ mitochondrial ratio of cytochrome $c(4.1 \pm 0.19$-fold $)$ during hypertrophy compared with control or Ml. Although the expression of Bax increased significantly during $\mathrm{MI}$ (2.51 \pm 0.08-fold) compared with control, cytosolic/mitochondrial ratio of cytochrome $\mathrm{c}$ decreased significantly compared with either hypertrophy or control (Figure 3a; Supplementary Figure S2). Further corroboration by immunofluorescence studies in vitro in Angll-treated cardiomyocytes showed pronounced upregulation of cytochrome $\mathrm{c}$ in the cytosol, compared with either control or hypoxic cardiomyocytes (Figure 3b). Among the Angll-treated cardiomyocytes, $43 \pm 0.9 \%$ cells showed an increased expression of cytosolic cytochrome c, whereas $<10 \%$ cells stained positive for cytosolic cytochrome $\mathrm{c}$ in case of hypoxic cardiomyocytes or control.

The role of VDAC1 as a chief regulator of cardiomyocyte apoptosis during hypertrophy was further confirmed by using VDAC1 siRNA treatment in vivo. Rats treated with VDAC1 siRNA during induction of hypertrophy showed marked decrease in the cytosolic/mitochondrial ratio of cytochrome c $(2.76 \pm 0.18$-fold) and significantly reduced activity of caspase 3 ( $2 \pm 0.22$-fold) compared with rats treated with non-specific siRNA. The siRNA treatment did not alter caspase 3 activity significantly during $\mathrm{MI}$ (Figures $3 \mathrm{c}$ and d).

Activation of GRP78-mediated ER stress pathway leads to induction of apoptosis during MI. As GRP78 - a marker for ER stress - was found to be exclusively upregulated during $\mathrm{MI}$, the expression status of ER stress pathway proteins was studied in the three groups. The expression of ER-resident PKR-like elF2 $\alpha$ kinase (PERK; $2.64 \pm 0.07$-fold) and phospho inositol-requiring enzyme 1 (IRE1) to IRE1 ratio (2.02 \pm 0.14 -fold) increased significantly during $\mathrm{Ml}$ compared with the other two groups (Figure 4a; Supplementary Figures S3A and B). Active cleavage product of activating transcription factor 6 (ATF6) and active X-Boxbinding protein 1 (XBP1), the downstream mediators of ER stress pathway were also found exclusively during MI (Figure 4a). Nuclear translocation of active ATF6 and XBP1 transcription factors, as assessed in vitro by immunofluorescence study, revealed ATF6-positive nuclei in $32 \pm 0.69 \%$ of hypoxic cardiomyocytes, whereas $31 \pm 0.91 \%$ nuclei were positive for XBP1. Less than $5 \%$ cells showed either ATF6- or XBP1-positive nuclei in case of either the hypertrophied cardiomyocytes or control cells (Figure $4 \mathrm{~b}$; Supplementary Figures S3 C and D).

Analysis of the ER stress-mediated apoptotic pathway in vivo by immunoblot analysis revealed that levels of activating transcription factor 4 (ATF4) (2.12 \pm 0.1 -fold), CCAAT/enhancer-binding protein homologous protein (CHOP; $2.7 \pm 0.07$-fold), tumor necrosis factor receptorassociated factor 2 (TRAF2; $2.32 \pm 0.06$-fold) and phospho c-Jun N-terminal kinase (JNK) to JNK ratio (1.72 \pm 0.08 -fold) increased significantly during $\mathrm{MI}$ compared with two other groups, although the level of total JNK remained unaltered in all groups. However, the expression of $\mathrm{Bcl}-2$ was found to be significantly downregulated (2.7 \pm 0.13 -fold) during $\mathrm{MI}$ compared with hypertrophy, though it was marginally higher $(1.17 \pm 0.22$-fold $)$ compared with control (Figure 4c; Supplementary Figure S4A-E). Upregulation of TRAF2 led to recruitment and subsequent cleavage of ERassociated caspase 12 exclusively during MI (Figure 4c). Nuclear translocation of active transcription factor $\mathrm{CHOP}$ scored by immunofluorescence study in vitro revealed $26 \pm 0.7 \%$ CHOP-positive nuclei among the hypoxic cardiomyocytes compared with $<2 \%$ among Angll-treated cardiomyocytes or control cells (Figure 4d; Supplementary Figure S4F). 

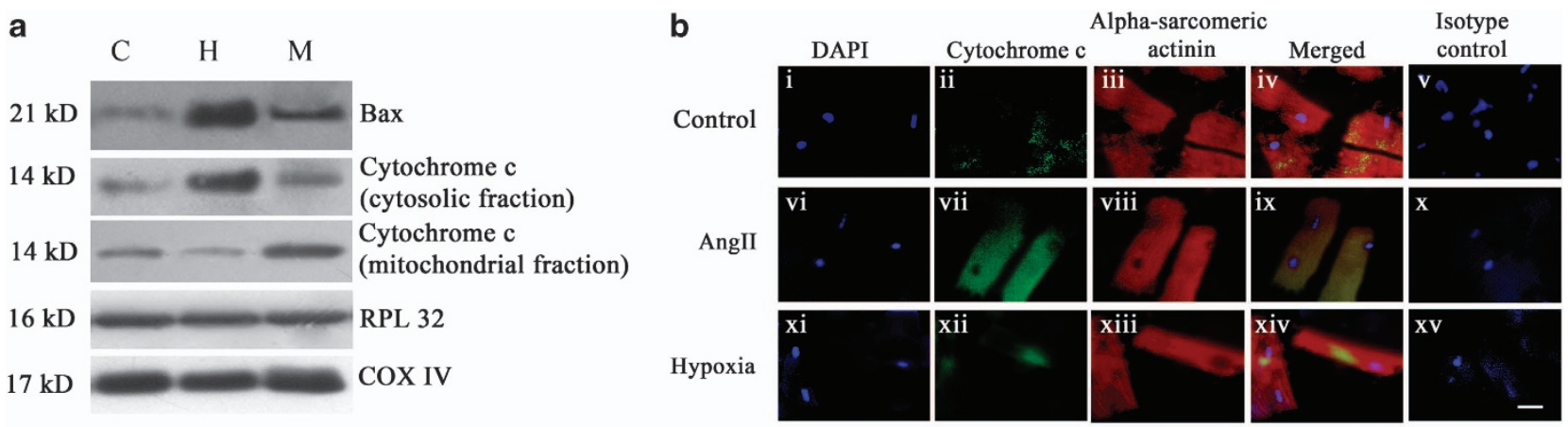

C
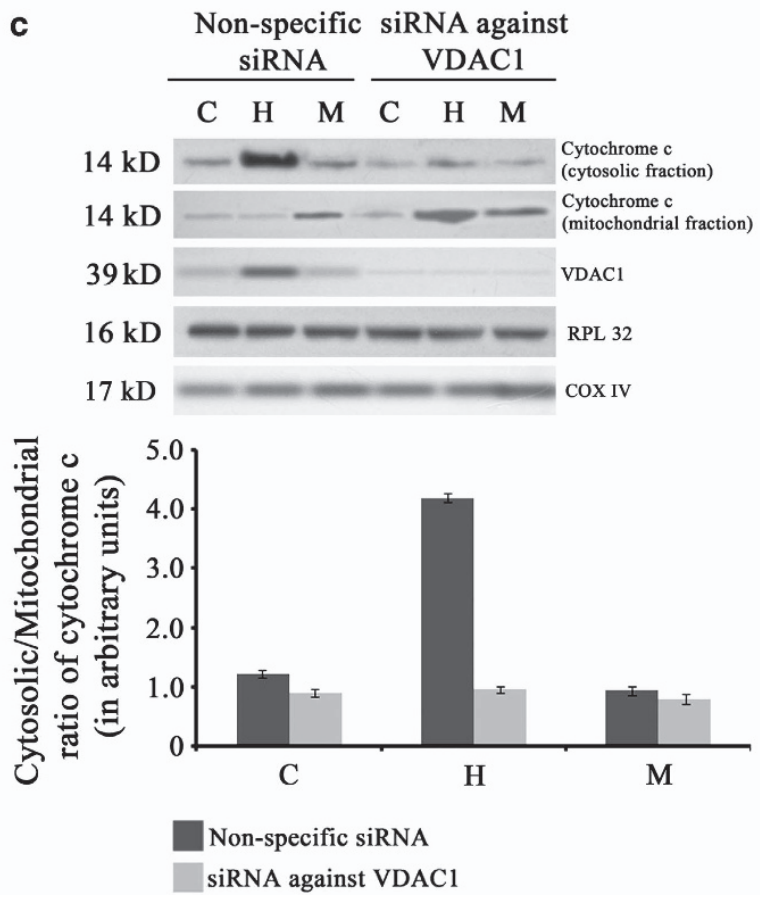

d

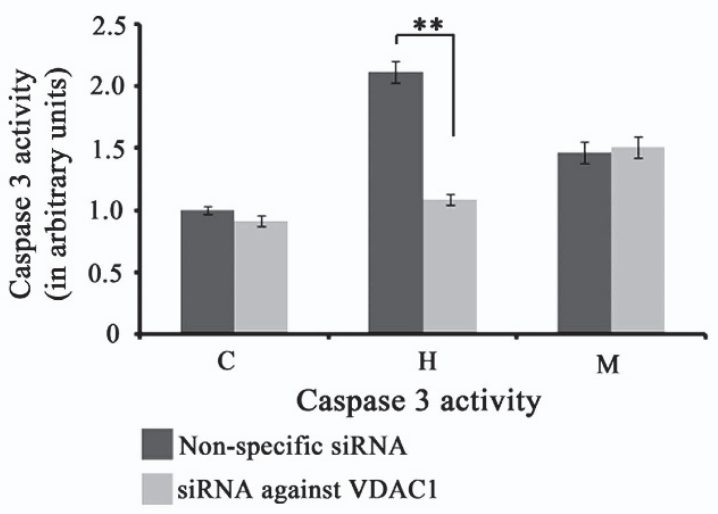

Figure 3 Activation of mitochondrial apoptotic machinery during hypertrophy. (a) Western blot analyses showing significant increase in the expression of Bax and cytochrome $c$ in hypertrophy $(\mathrm{H})$ compared with either MI (M) or sham control (C). RPL32 was used as loading control for cytosolic proteins whereas COX IV was used as loading control for mitochondrial proteins. Data is representative of three independent experiments. (b) Immunofluorescence study showing upregulation of mitochondrial apoptotic marker cytochrome $\mathrm{c}$ in hypertrophic cardiomyocytes. Adult cardiomyocytes were stained with antibody against cytochrome $\mathrm{c}$ (panel (i-iv), $(v i-i x),(x i-x i v))$ for three groups (Control, Ang II-treated cells and hypoxic cardiomyocytes). Pronounced expression of cytochrome c (green fluorescence) was observed in Ang II-treated cells only compared with either hypoxic or untreated control. Cells were counter stained with sarcomeric $\alpha$-actinin antibody for cardiomyocyte specificity. Panel $(\mathrm{v}),(\mathrm{x})$ and $(\mathrm{xv})$ represent isotype control images. (Scale bar $=10 \mu \mathrm{m})(\mathrm{c})$ Western blot analyses showing successful knockdown of VDAC1 and significant decrease in the level of cytosolic cytochrome $c$ in rats treated with VDAC1 siRNA during hypertrophy. This siRNA treatment had no effect on the level of cytosolic cytochrome $c$ during M. (d) Graph showing caspase 3 activity in the three experimental groups (C, H and M) treated with either siRNA against VDAC1 or non-specific siRNA. Significant decrease in caspase 3 activity occurred in rats treated with VDAC1 siRNA during hypertrophy. The siRNA treatment had no effect on caspase 3 activity during MI. $\left({ }^{\star *} P<0.01\right)$

Furthermore, the predominance of ER stress-induced apoptosis during $\mathrm{Ml}$ was confirmed by chemical chaperone tauroursodeoxycholic acid (TUDCA) treatment in MI rats showing marked downregulation of ER stress-mediated apoptotic markers CHOP $(2.3 \pm 0.21$-fold $)$ and phospho JNK to JNK ratio ( $1.51 \pm 0.12$-fold) (Figures $5 a$ and b). TUDCA treatment also resulted in significantly reduced cleavage of caspase 12 (Figure 5a) and decreased caspase 3 activity (3.1 \pm 0.25 -fold) during Ml compared with animals treated with vehicle. TUDCA treatment had no effect on caspase 3 activity during hypertrophy (Figure 5c).
Increased phosphorylation and translocation of CRYAB to mitochondria inhibits cytochrome $c$ release during MI. Proteomic analysis and subsequent immunoblotting revealed significant upregulation of CRYAB, a sHSP, during $\mathrm{MI}$ compared with hypertrophy or control (Figure 1). As, phosphorylation of CRYAB (at serine-59) has been reported to be critical for its activation and translocation to mitochondria and microfilaments, ${ }^{17}$ phosphorylation status of CRYAB was checked by immunoblot analysis in the three groups. Significantly higher phosphorylation of CRYAB (at serine-59) was recorded in both isoproterenol-treated $(2.79 \pm 0.15$-fold $)$ 
a

b

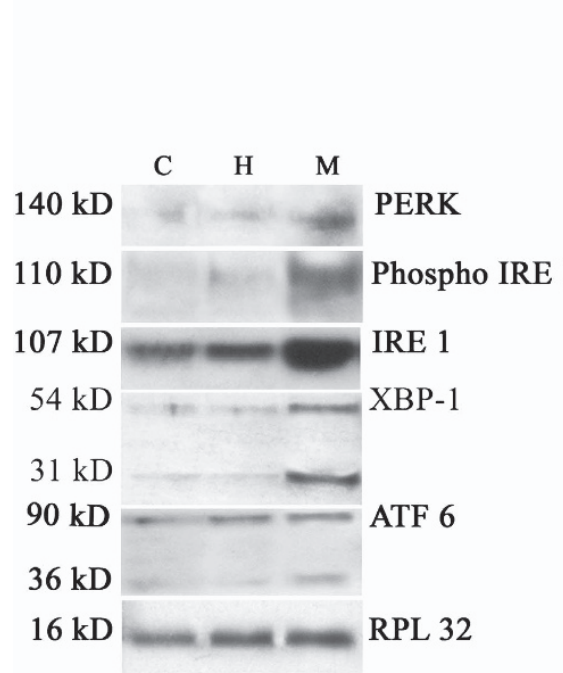

c

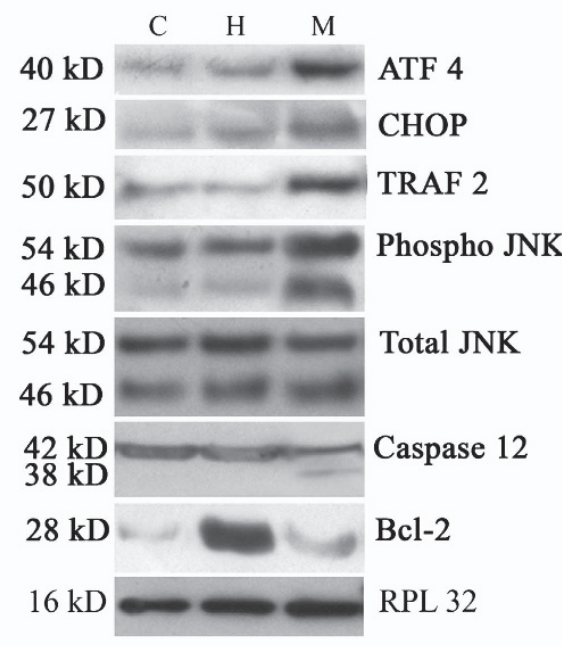

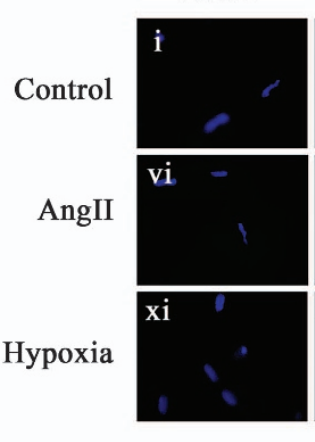

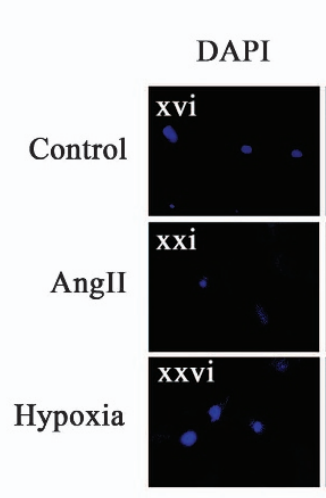

d

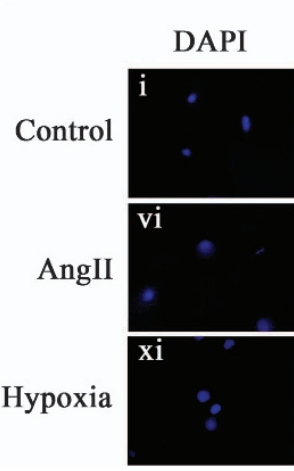

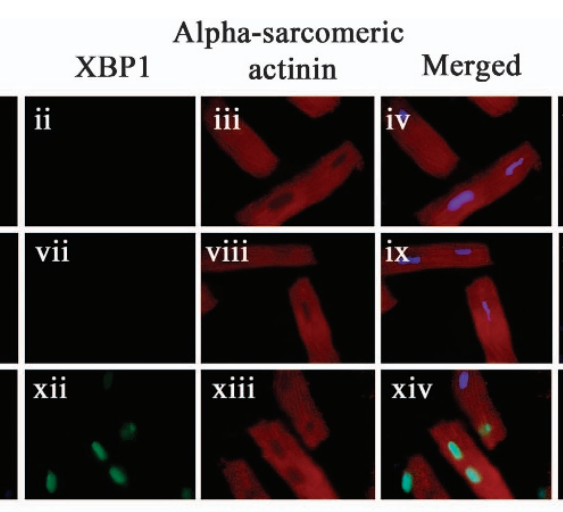

Isotype control

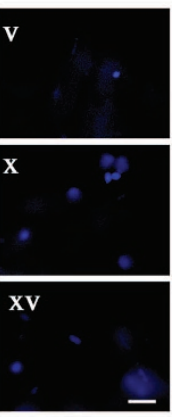

Alpha-sarcomeric

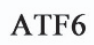
actinin

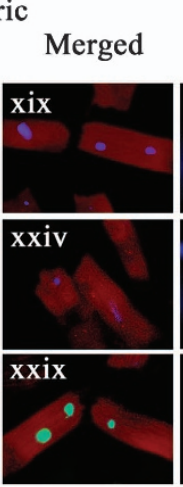

Isotype control
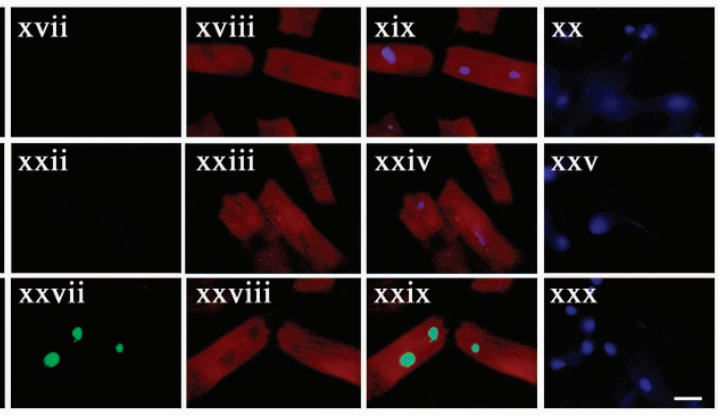

Isotype control

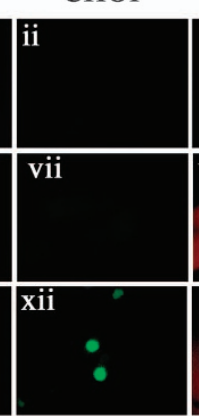

Alpha-sarcomeric

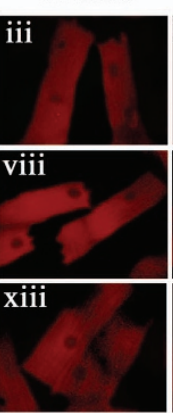

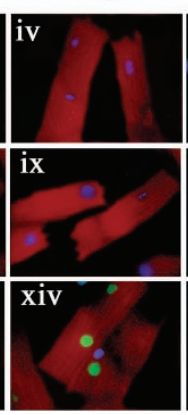

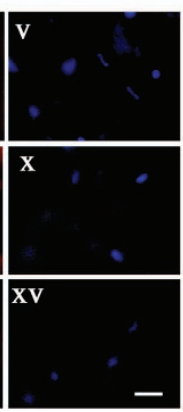

Figure 4 Activation of ER stress pathway and ER stress-induced apoptosis during MI. (a) Immunoblot analyses showing increased expressions of PERK, IRE 1 and phospho IRE 1 during MI (M) in vivo compared with either hypertrophy (H) or control (C). Immunoblot analyses also showed cleavage of ATF6 (36 kDa) and active XBP1 $(31 \mathrm{kDa})$ exclusively during Ml that was absent either in hypertrophy or control. RPL32 was used as internal control. Data is representative of three independent experiments. (b) Immunofluorescence study showing nuclear translocation of XBP1 and ATF6 in hypoxic cardiomyocytes in vitro. Adult cardiomyocytes were stained with antibodies against XBP1 (panel (i-iv), (vi-ix), (xi-xiv)) and ATF 6 (panel (xvi-xix), (xxi-xxiv), (xxvi-xxix)). Nuclear translocation of both XBP1 and ATF6 was observed in hypoxic cardiomyocytes only (green fluorescence) that was absent in Angll-treated myocytes and untreated control. Panel $(\mathrm{v}),(\mathrm{x}),(\mathrm{xv}),(\mathrm{xx}),(\mathrm{xxv})$ and (xxx) represent isotype control images. (c) Immunoblot analyses showing significant increase in the expressions of ATF4, CHOP and TRAF 2 and ratio of phospho JNK to JNK during M compared with either H or C. Expression levels of total JNK remained unaltered in all the three groups. Expression of Bcl-2 protein was significantly downregulated during $\mathrm{M}$ compared with hypertrophy. Immunoblot analyses also showed cleavage of active caspase $12(38 \mathrm{kDa})$ exclusively during $\mathrm{M}$. Data is representative of three independent experiments. (d) Immunofluorescence study showing nuclear translocation of $\mathrm{CHOP}$ in hypoxic cardiomyocytes. Nuclear translocation of $\mathrm{CHOP}$ (green fluorescence) was observed in hypoxic cardiomyocytes only. Translocation was found to be absent in Angll-treated myocytes or untreated control. Cardiomyocyte specificity was confirmed by counterstaining cells with sarcomeric alpha actinin antibody. Panel (v), (x), (xv) represent isotype control images. (Scale bar $=10 \mu \mathrm{m})$

and LAD-ligated (2.92 \pm 0.21 -fold) heart samples compared with either hypertrophy or control (Figure 6a). Immunoelectron microscopy also revealed increased translocation of phospho CRYAB protein to the mitochondrial surface of myocytes during $\mathrm{Ml}$ compared with hypertrophy (Figure 6b). We wanted to further check whether phospho CRYAB binding to VDAC1 has any role in bypassing the mitochondrial apoptotic pathway in cardiomyocytes during MI. Coimmunoprecipitation experiment revealed significant increase in binding of phospho CRYAB with VDAC1 protein during $\mathrm{MI}$ in both isoproterenol-treated (2.67 \pm 0.21 -fold) and LAD-ligated (2.86 \pm 0.24 -fold) heart samples compared with 

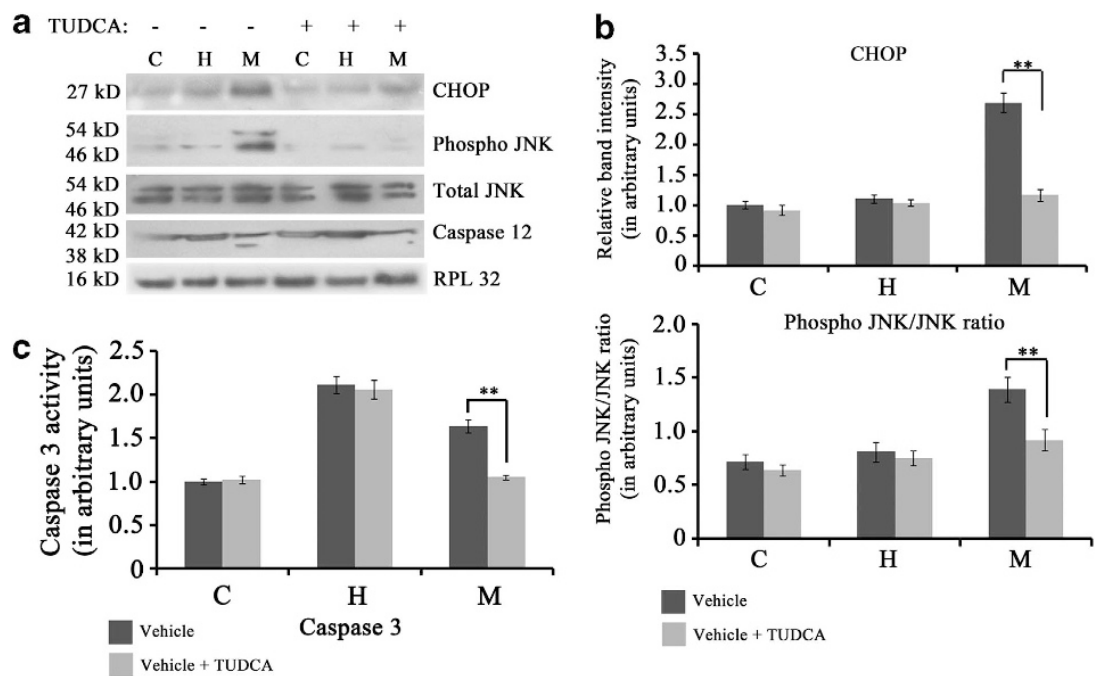

Vehicle + TUDCA

Figure 5 (a) Immunoblot analyses showing significant decrease in the level of CHOP, ratio of phospho JNK to JNK and cleavage of caspase12 in rats treated with TUDCA during MI. (b) Graph showing relative band intensity of CHOP and phospho JNK in the three experimental groups viz., control $(\mathrm{C})$, hypertrophy $(\mathrm{H})$ and $\mathrm{MI}(\mathrm{M})$ treated with either TUDCA or vehicle alone. (c) Graph showing relative caspase 3 activity in $\mathrm{C}, \mathrm{H}$ and $\mathrm{M}$ groups treated with either TUDCA or vehicle alone. Significant decrease in caspase 3 activity occurred in TUDCA-treated rats during $M$ with no effect during hypertrophy. $\left.{ }^{* \star} P<0.01\right)$
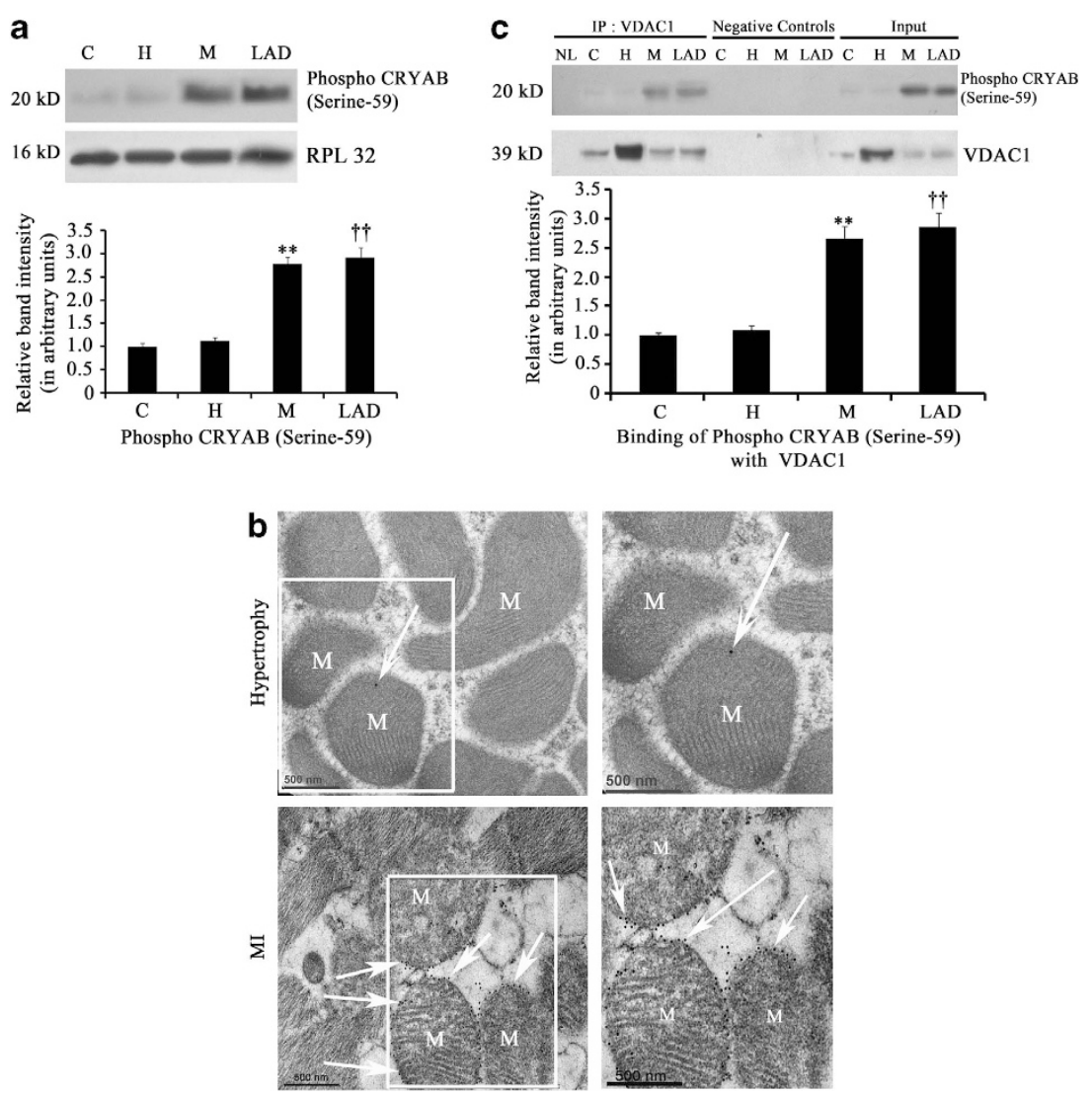

Figure 6 (a) Immunoblot analysis showing significant increase in the expression of phospho CRYAB (serine-59) in the MI group (isopreternol-treated (M) and LAD-ligated $(L A D)$ ) in vivo compared with either hypertrophy $(H)$ or sham control $(C)$. RPL32 was used as internal control. Data is representative of three independent experiments. $\left({ }^{*} P<0.01\right.$ with respect to $M$ versus $H$ and $M$ versus $C$; $\dagger \dagger P<0.01$ with respect to $L A D$ versus $H$ and $L A D$ versus $\left.C\right)$. (b) Immunoelectron microscopy studies showing pronounced presence of phospho CRYAB (serine-59) particles on mitochondrial (M) surface of Ml samples compared to hypertrophy (Scale bar $=500 \mathrm{~nm}$ ). (c) Coimmunoprecipitation study followed by immunoblotting showing significantly increased binding between phospho CRYAB (serine-59) and VDAC1 protein during MI (isopreternol-treated $(M)$ and LAD-ligated $(L A D))$ compared with $H$ or $C\left({ }^{* *} P<0.01\right.$ with respect to $M$ versus $H$ and $M$ versus $C$; $\dagger \dagger P<0.01$ with respect to $L A D$ versus $\mathrm{H}$ and $\mathrm{LAD}$ versus $\mathrm{C})$ 


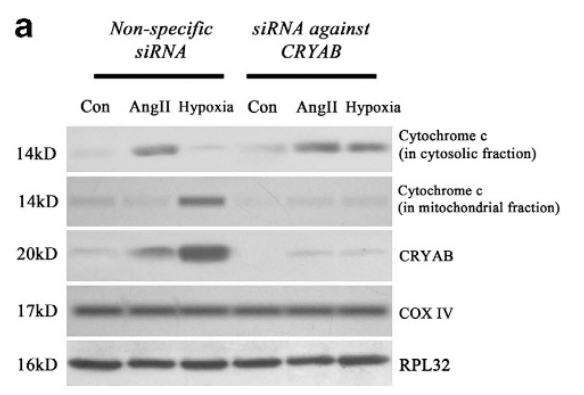

b
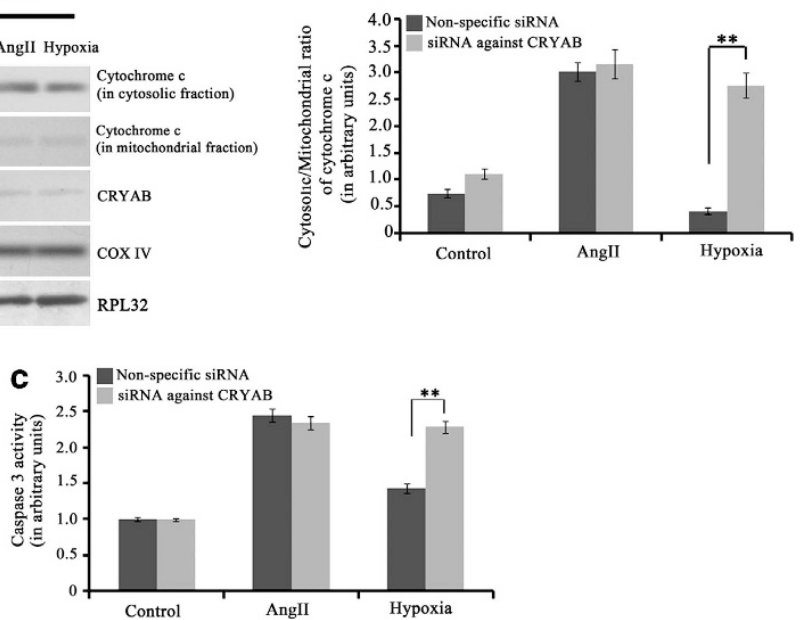

Figure 7 (a) Immunoblot analysis showing successful knockdown of CRYAB and significant increase in the ratio of cytosolic/mitochondrial cytochrome $\mathrm{c}$ in myocytes transfected with siRNA against CRYAB during induction of hypoxia. This siRNA treatment had no effect on the level of cytosolic cytochrome $c$ in myocytes treated with Ang II. (b) Graph showing ratio of cytosolic/mitochondrial cytochrome $c$ in control, Angll-treated or hypoxic myocytes transfected with either siRNA against CRYAB or non-specific siRNA ( $\left.{ }^{* *} P<0.01\right)$. (c) Graph showing caspase 3 activity in the three experimental groups (Control, Ang II and hypoxia) treated with either siRNA against CRYAB or nonspecific siRNA. Significant increase in caspase 3 activity was observed in hypoxic cardiomyocytes treated with siRNA against CRYAB. The siRNA treatment had no effect on caspase 3 activity during hypertrophy $\left({ }^{*} P<0.01\right)$

control or hypertrophy (Figure 6c). Further, mass spectrometric analysis of the protein pool that was immunoprecipitated by phospho CRYAB antibody revealed that, among other proteins, adenine nucleotide translocator (ANT), which forms cytochrome c release gateway along with VDAC1, was found exclusively in $\mathrm{MI}$ samples (Supplementary Table S1). This suggests that activated CRYAB protein binds to VDAC1 channel, resulting in inhibition of release of cytochrome $\mathrm{c}$ into cytosol during $\mathrm{MI}$. This was further confirmed by transfection of cardiomyocytes with CRYAB-specific siRNA. The siRNA-transfected hypoxic cardiomyocytes showed significant increase in the cytosolic/ mitochondrial ratio of cytochrome c $(2.82 \pm 0.21$-fold; Figures $7 \mathrm{a}$ and $\mathrm{b})$ and caspase 3 activity (1.6 \pm 0.09 -fold) compared with cells treated with non-specific siRNA (Figure 7c). CRYAB siRNA treatment had no effect either on levels of cytosolic cytochrome $\mathrm{c}$ or caspase 3 activity in either hypertrophic cardiomyocytes or control cells.

\section{Discussion}

It is now well known that cardiomyocyte apoptosis has an important role in the progression of heart failure. ${ }^{3,6}$ However, varied signaling molecules responsible for myocyte apoptosis in etiologically different cardiac diseases has not yet been addressed. Our study is the first of its kind where differential modes of myocyte apoptosis were deciphered during two etiologically different cardiac diseases viz., cardiac hypertrophy and $\mathrm{MI}$, and $\mathrm{CRYAB}$ protein was also identified as a key player regulating the differential apoptotic mechanism in diseased cardiomyocytes during these two cardiac disease forms.

The cardiac hypertrophy models used in our study, both in vivo and in vitro, were previously established with significant increase in hypertrophy marker genes and severely compromised cardiac function. ${ }^{3}$ Successful generation of the in vivo and in vitro $\mathrm{Ml}$ models were substantiated by significantly induced expression of xanthine oxidase, a wellknown marker of ischemia, earlier detected in cardiac myocytes and coronary endothelial cells of patients with ischemic heart disease. ${ }^{18}$ As it is known that isoproterenolinduced $\mathrm{Ml}$ in vivo occurs because of relative hypoxia as a result of increase in the double product (blood pressure $x$ heart rate), ${ }^{19}$ an in vitro study was undertaken to check the effect of hypoxic stress on adult cardiomyocytes.

The established apoptotic markers like caspase 3 and PARP were found to be cleaved (Figure 2a), and caspase 3 activity (Figure $2 b$ ) as well as the number of TUNEL-positive nuclei (Figure 2c) were increased during both hypertrophy and $\mathrm{Ml}$ in our study, signifying apoptosis as a hallmark of compensated cardiac function. ${ }^{3}$ However, the proteomic analysis indicated upregulation of molecules involved in distinct signaling pathways, leading to cardiomyocyte death in these two forms of cardiac diseases.

Our data revealed that VDAC1, a mitochondrial outer membrane channel protein, along with Bax and cytosolic cytochrome $\mathrm{c}$ was upregulated exclusively during hypertrophy. Increased VDAC1 mRNA level has been reported earlier in left ventricular septal tissue of human patients suffering from long-standing hypertrophic cardiomyopathy. ${ }^{20}$ VDAC1, activated by the proapoptotic protein Bax, ${ }^{21}$ forms a gateway for the release of cytochrome $c$ from mitochondrial intermembrane space to cytosol along with ANT and cyclophilin D. ${ }^{22}$ However, the change in the expression of VDAC1 and cytosolic cytochrome $\mathrm{c}$ was negligible during $\mathrm{Ml}$ compared with control, although it was interesting to note that the level of cytochrome $\mathrm{c}$ in mitochondrial fraction during $\mathrm{Ml}$ was high (Figure 3a). Silencing of VDAC1 in vivo showed significant decrease in caspase 3 activity during hypertrophy, but had no significant effect during MI. Thus, although apoptotic 


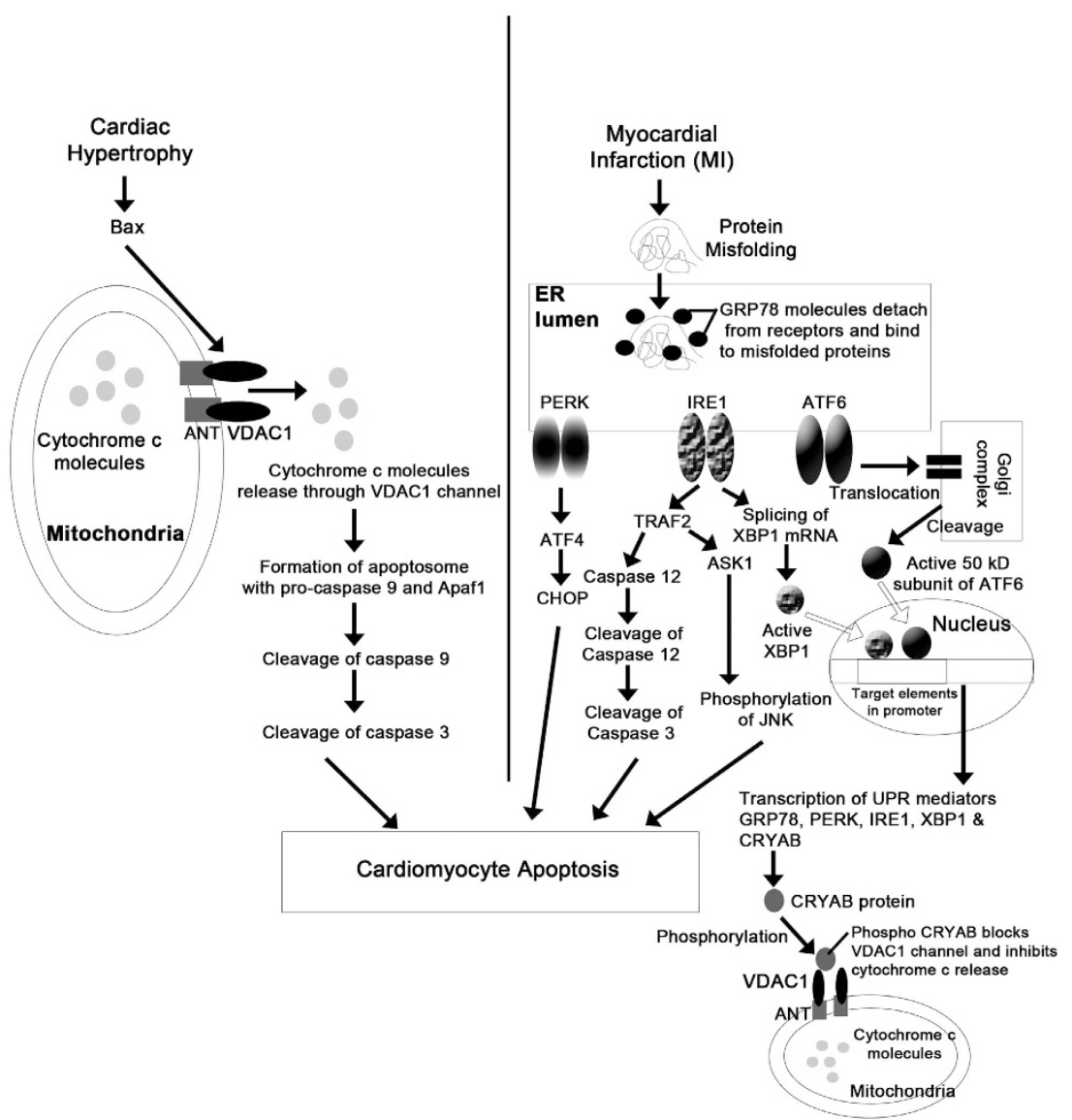

Figure 8 Schematic diagram showing two distinct routes of cardiomyocytes apoptosis involving two different cellular organelles - mitochondria and ER during cardiac hypertrophy and $\mathrm{MI}$, respectively, along with CRYAB as a regulator for the apoptotic programming in these two cardiac disease forms.

machinery is induced during both hypertrophy and $\mathrm{MI}$, the mitochondrial pathway involving VDAC1 is predominant during cardiac hypertrophy.

Our study also revealed that GRP78 - an ER-resident protein - assisting in protein folding and the most important upstream regulator of the unfolded protein response due to ER-stress, ${ }^{23}$ was exclusively upregulated during MI (Figure 1) along with the levels of proximal effectors of ER stress ${ }^{23}$ PERK, IRE1 and phospho IRE1 (Figure 4a). Active cleaved subunit of ATF6 and functional product of XBP1 also appeared exclusively during $\mathrm{MI}$ with nuclear translocation of these two proteins in hypoxic cardiomyocytes. Although there are reports of ER stress during long-term cardiac hypertrophy, ${ }^{24}$ our results did not reveal any significant change in the levels of these ER stress markers during hypertrophy compared with control, in accordance with Sari et al. ${ }^{25}$

Further, exclusive upregulation of the major ER stressinduced apoptotic mediators ${ }^{23}$ ATF4, CHOP and TRAF2 in MI hearts and nuclear translocation of $\mathrm{CHOP}$ in the hypoxic cardiomyocytes signifies induction of ER stress-mediated apoptosis during MI (Figures $4 a-d)$. CHOP has been reported to induce proapoptotic proteins like growth arrest and DNA damage-inducible protein $34, \mathrm{Bim}$ and Tribbles-related protein-3 but downregulates antiapoptotic $\mathrm{Bcl}-2$ protein. ${ }^{23}$ Significant upregulation of phosphorylated JNK during MI was observed in our study compared with the two other groups. It was reported earlier that, TRAF2 recruited by phospho IRE1, binds to apoptosis signal-regulating kinase 1 , and initiates the MAPK cascade to activate the apoptotic mediator JNK during severe ER stress. ${ }^{26}$ Active JNK is also known to induce apoptosis via activation of proapoptotic cJun or $\mathrm{Bcl}-2$ associated death promoter protein and inactivation of antiapoptotic protein $\mathrm{Bcl}-2{ }^{27}$ Further, it is known that recruitment and activation of caspase 12 by either caspase 7 or TRAF2 can mediate the activation of caspase 3 without the involvement of mitochondria. ${ }^{28}$ Our data revealed cleavage of caspase 12 during $\mathrm{MI}$, that was absent in the two other groups (Figure 4c). Activation of all these proteins signifies the induction of myocyte apoptosis during $\mathrm{Ml}$ as a result of severe ER stress beyond a certain threshold level. Although, induction of ER stress and ATF4-CHOPmediated apoptosis was reported earlier in a mouse model of $\mathrm{MI}^{29}$ our study revealed involvement of other ER stressmediated apoptosis signaling such as JNK pathway and caspase 12 pathway during MI that remained unaltered during hypertrophy. This finding was reinforced by significant downregulation of ER stress-induced apoptotic markers in MI rats treated with chemical chaperon TUDCA ${ }^{30}$ (Figures 5a-c).

Thus, our study revealed that among other altered proteins, VDAC1 orchestrates mitochondrial Bax-cytochrome c pathway-regulated cardiomyocyte apoptosis during hypertrophy and ER-mediated myocyte apoptosis induced by GRP78, was 
activated in case of MI (Figure 8). It seemed to be a paradox that even though Bax, an important proapoptotic regulator of mitochondrial pathway, was upregulated and level of mitochondrial cytochrome $\mathrm{c}$ also increased during $\mathrm{MI}$, no change in the level of cytosolic cytochrome $c$ was observed. In this study, we found significant upregulation of CRYAB, which after phosphorylation (at serine-59) has been reported to translocate to mitochondria and have antiapoptotic role during I/R injury. ${ }^{17}$ It has been shown to bind with VDAC1 during hypoxic stress induced by $\mathrm{H}_{2} \mathrm{O}_{2}$ in neonatal mouse cardiomyocytes. ${ }^{31}$ On the basis of these earlier reports, we tried to seek a possible explanation to this paradox by studying the role of CRYAB during MI. Increased phosphorylation of CRYAB (at serine-59) indicated increased activation of CRYAB during MI, whereas immunoelectron microscopy and coimmunoprecipitation experiments confirmed mitochondrial translocation and binding of CRYAB to both VDAC1 and ANT exclusively during MI (Figures 6a-c; Supplementary Table $\mathrm{S1}$ ). This finding led us hypothesize that during MI, CRYAB, which is a downstream mediator of unfolded protein response, ${ }^{32}$ gets phosphorylated (at serine-59), translocates to mitochondria and in turn binds to VDAC1 channel, thus inhibiting cytochrome $c$ release into cytosol. The role of CRYAB was confirmed as in the presence of CRYAB siRNA mitochondrial pathway was activated in hypoxic cardiomyocytes, as evident by increased cytosolic cytochrome c (Figures $7 a-c)$. These findings clearly suggest that activated CRYAB acts as an inhibitor of mitochondrial apoptotic pathway during MI but not during hypertrophy.

Thus, this study clearly demonstrates that although apoptosis is a hallmark of both cardiac hypertrophy and MI, the molecular mechanisms leading to myocyte death are distinct in these two disease forms and two different organelles - mitochondria and ER have predominant roles in this process via VDAC1 and GRP78, respectively (Figure 8). This study also establishes the fact that CRYAB is a major molecular regulator of cardiomyocyte apoptosis, which blocks VDAC1 channel in MI samples, and is thus responsible for the shift of cardiomyocyte apoptotic signaling from mitochondria to ER during MI.

\section{Materials and Methods}

Animals used. Wistar rats used in this study were procured from the National Institute of Nutrition, Hyderabad, AP, India. The investigation conforms to the Guidelines for the Care and Use of Laboratory Animals published by the US National Institute of Health (NIH Publication no. 85-23, revised 1996) and was also approved by the Institutional Animal Ethics Committee, University of Calcutta (Registration no. 885/ac/05/CPCSEA), registered under 'Committee for the Purpose of Control and Supervision of Experiments on Laboratory Animals' (CPCSEA), Ministry of Environment and Forests, Government of India.

Generation of cardiac hypertrophy and MI in vivo. Twenty-fourweek-old male Wister rats $(n=10)$ were used to generate left ventricular hypertrophy by ligating their right renal artery for 14 days, and hypertrophy was measured as described previously. ${ }^{3} \mathrm{MI}$ was generated in 24-week-old male Wister rats $(n=10)$ by intraperitoneal injection of synthetic catecholamine isoproterenol hydrochloride (100 mg/kg body weight per day; Sigma-Aldrich, MO, USA), a $\beta$ adrenergic agonist, for two consecutive days as described previously. ${ }^{33}$ Isoproterenol treatment is the maximally studied and standardized non-invasive method for MI generation, as it mimics acute phase of MI in human patients. ${ }^{33}$ Similarly, a ligation model of MI was also generated by occlusion of left anterior descending coronary artery as described earlier with slight modifications ${ }^{34}$, and the animals were euthanized after 10 days of ischemic period. The age-matched sham-operated control underwent similar procedure without actual ligation of the artery or injection of isoproterenol hydrochloride.

Rats were anesthetized with an intraperitoneal injection of a combination of $100 \mathrm{mg} / \mathrm{kg}$ ketamine and $10 \mathrm{mg} / \mathrm{kg}$ xylazine, and were euthanized via intravenous ketamine injection at the end of the experiment. The infarct zone was identified in MI samples by TTC method, as described previously. ${ }^{35}$ The infarct area was measured using Image Tool software v2.0 (UTHSC, San Antonio, TX, USA). For subsequent protein and RNA extraction, cardiac tissue samples from all groups were collected and preserved in liquid nitrogen. Tissue was specifically collected from the infarct zone of the Ml group.

In vivo treatment with VDAC1 siRNA and ER stress inhibitor. siRNA against VDAC1:S136016/S136017(Catalogue no. 4457308, Ambion, Life Technologies, Grand Island, NY, USA) or non-specific siRNA (Catalogue no. 4457289, Ambion, Life Technologies) in $1 \times$ phosphate-buffered saline (PBS) was injected in ventricles of aortic-ligated rats $(n=5)$ at a concentration of 10 nmoles following manufacturer's protocol for last 10 days of experimentation, as described earlier. $^{3}$ Control and MI samples were also treated with the same siRNA against VDAC1 or non-specific siRNA during the experimental period.

TUDCA, an ER stress inhibitor and a chemical chaperone, was administered $(50 \mathrm{mg} / \mathrm{ml}, 400 \mathrm{mg} / \mathrm{kg}$, i.v.) to control, hypertrophic and infarcted rats $(n=5)$ during the experimental period, as described earlier. ${ }^{36}$

Generation of cardiomyocyte hypertrophy and ischemia in vitro. Adult myocytes were isolated from hearts of 20-week-old male Wister rats, following the procedure described previously. ${ }^{37}$ Approximately $90 \%$ pure isolated cardiomyocytes were confirmed by staining with sarcomeric $\alpha$-actinin antibody (Abcam, Cambridge, MA, USA). In vitro cardiomyocyte hypertrophy was generated by incubating the cells in absence (control) or presence (treated) of $10^{-8} \mathrm{~mol} / /$ (Sar1) Angll; Bachem, Torrance, CA, USA) for $24 \mathrm{~h}$ at $37^{\circ} \mathrm{C} .^{37}$

Ischemic condition was generated by subjecting cultured adult cardiomyocytes to hypoxic condition as described earlier, with slight modifications ${ }^{38}$ using Galaxy $170 \mathrm{R}$ incubator (New Brunswick, Edison, $\mathrm{NJ}, \mathrm{USA}$ ) maintained at $1 \% \mathrm{O}_{2}$ and $5 \% \mathrm{CO}_{2}$ for $5 \mathrm{~h}$. Cardiomyocytes were maintained in glucose and serum-free DMEM (Gibco, Carlsbad, CA, USA) during hypoxic tenure.

Treatment of adult cardiomyocytes with siRNA against alpha crystallin B (CRYAB). Adult cardiomyocytes were transfected with either CRYAB-specific siRNA (Catalogue no. SI01501913, Qiagen, Valencia, CA, USA) to block the expression of CRYAB or negative control siRNA (AllStars Negative Control siRNA, Catalogue no. 1027280,Qiagen) using HiPerFect Transfection Reagent (Qiagen), as per manufacturer's protocol.

Reverse transcriptase-PCR. Total RNA was isolated from cardiac ventricular tissues and isolated adult cardiomyocytes using TRIzol reagent (Invitrogen, Carlsbad, CA, USA). Reverse transcription was done using Cloned AMV First-Strand cDNA Synthesis Kit (Invitrogen). Expression of hypertrophy marker genes ANF and $\beta$-MHC was studied, as described previously. ${ }^{3}$

Protein extraction. After the experimental period, hearts were dissected out and perfused in chilled PBS. Then the heart tissues were homogenized in protein extraction buffer $(7 \mathrm{M}$ urea, $4 \%$ (w/v) CHAPS, $2 \mathrm{M}$ thiourea and $1 \mathrm{X}$ EDTA - free protease inhibitor cocktail (Roche, South San Francisco, CA, USA)) using Dounce homogenizer for $30 \mathrm{~min}$. The tissue lysates were then subjected to centrifugation at 12500 r.p.m. at $4^{\circ} \mathrm{C}$ for $20 \mathrm{~min}$. The supernatants were collected from each sample and concentrations of protein were estimated by Bradford assay. Protein from adult cardiomyocytes was isolated using M-PER Mammalian protein extraction reagent (Thermo Scientific, Rockford, IL, USA). Mitochondrial fraction from either cell or tissue samples were isolated by differential centrifugation method, as described earlier. ${ }^{39}$

Western blotting. Twenty micrograms of total protein extract from each of the three groups - control, hypertrophy and MI - was fractionated by SDS-PAGE and transferred to $\mathrm{PVDF}^{+}$membrane (Millipore, Billerica, MA, USA), followed by incubation with monoclonal antibodies to Bax (BD Pharmingen, Sparks, MD, USA), VDAC1, ATF6; Abcam), cytochrome c, PERK, CHOP, JNK, phospho JNK, caspase 3 (Cell Signaling, Danvers, MA, USA), polyclonal antibodies to xanthine oxidase, XBP1(Santa Cruz, Santa Cruz, CA, USA), GRP78, IRE1, phospho IRE1, 
ATF4, TRAF2, caspase 12, CRYAB, phospho CRYAB (Abcam), PARP, B-cell lymphoma 2 (Cell Signaling) and HRP-conjugated secondary antibodies (Pierce, Rockford, IL, USA). Immunoreactive bands were visualized using Immobilon Western chemiluminescence HRP substrate (Millipore). 60S ribosomal protein L32 (RPL32; Abcam) was used as loading control for cytosolic proteins and COX IV was used as loading control for mitochondrial proteins. The blots were scanned and quantitated using GeIDoc XR system and Quantity One software version 4.6.3 (Bio-Rad, Hercules, CA, USA). Three independent experiments were performed with five biological replicates for each group of animals.

TUNEL staining. TUNEL staining of paraffin-embedded tissue sections from all the experimental groups was performed with the In Situ cell death detection kit (Roche, Mannheim, Germany), as per manufacturer's instruction. Negative controls (without any apoptotic inducer or TdT enzyme) and positive controls (Dnase l-treated) were also included in the experiment. TUNEL-positive cells were visualized under fluorescent microscope (Olympus BX51, Progres C5, Center Valley, PA, USA).

Immunofluorescence studies. Cardiomyocytes were stained with monoclonal antibodies to ATF6 (Abcam), cytochrome c, CHOP (Cell Signaling), polyclonal antibodies to XBP1 (Santa Cruz) and sarcomeric $\alpha$-actinin (Abcam), followed by incubation with labeled secondary antibodies (Alexa fluor 488, Alexa fluor 594, Alexa fluor 633; Molecular Probes, Eugene, OR, USA), as described earlier. ${ }^{40}$ After mounting with Vectashield (with DAPI; Vector Laboratories, Burlingame, CA, USA), cells were visualized under fluorescent microscope (Olympus BX51, Progres C5). For each experimental condition, isotype controls were included.

Coimmunoprecipitation and mass spectrometry. Coimmunoprecipitation was done as described earlier ${ }^{3}$, following manufacturer's protocol (Pierce Co-Immunoprecipitation Kit, ThermoFisher, Waltham, MA, USA). After immunoprecipitating with VDAC1 antibody (Abcam), western blotting was done using polyclonal antibodies against CRYAB and phospho CRYAB (Abcam), as described in the previous section. Equal protein loading was confirmed by probing with monoclonal antibody to VDAC1 (Abcam).

For mass-spectrometric analysis, cardiac protein from all the three groups was immunoprecipitated with polyclonal antibody to phospho CRYAB (Abcam). The immunoprecipitated proteins were eluted with soft elution buffer, as described earlier. ${ }^{41}$ Elutes were then digested and peptide data was acquired on Prominence UFLC system (Shimadzu, Tokyo, Japan) coupled to the 5600 TripleTof fitted with the Duospray Ion Source in positive polarity with high resolution MS scan (m/z 350-1600) followed by 12 high-resolution MS/MS Scans (precursors charge state 2-5) in information-dependent acquisition mode. For specificity, the protein samples were pre-cleared with rabbit IgG (Invitrogen, Grand Island, NY, USA).

Immunoelectron microscopy. Fixation and embedding of cardiac tissue samples were performed as described earlier. ${ }^{42}$ Thin sections $(80-90 \mathrm{~nm})$ were incubated with polyclonal antibody against phospho CRYAB (ser-59; Abcam), followed by incubation with anti-rabbit secondary antibody conjugated with colloidal gold (BBI, Cardiff, UK). After brief wash, the sections were stained with $2.5 \%$ uranyl acetate, and visualized under Morgagni 268D transmission electron microscope (Fei Company, Eindhoven, The Netherlands).

Caspase 3 protease activity assay. Caspase 3 activity from cardiac tissue or adult cardiomyocytes from all experimental groups was determined using ApoAlert caspase 3 Fluorescent Assay Kit (Clontech Laboratories, Mountain View, CA, USA). ${ }^{3}$

Determination of cardiac function. Two-dimensional echocardiography was performed to determine cardiac function in vivo, as described previously. ${ }^{3}$ Briefly, cardiac function of lightly sedated rats from all experimental groups (control, hypertrophy and MI) were evaluated using M-mode views to assess the LVDD and \%FS. Digitized images were obtained using an ultrasound system (Vivid S5 system, GE Healthcare, Milwaukee, WI, USA).

Statistical analysis. Results were expressed as mean \pm S.E. of $>3$ independent experiments. Data was analyzed by ANOVA using SPSS (v13.0; IBM, New York, NY, USA). Values of $P \leqslant 0.05$ were considered as significant.

\section{Conflict of Interest}

The authors declare no conflict of interest.
Acknowledgements. This work was funded by Grants from Council of Scientific and Industrial Research (Grant number 37(1393)/10/EMR-II), India; Department of Science and Technology (Grant number F.No.SR/SO/HS-100/2009), India and University Grants Commission (Grant number F.No.39-108/2010 (SR)), India to S Sarkar. A Mitra was supported by fellowship from University Grants Commission. Part of this work was funded by CSIR network project (NWP004) Plasma proteomics: health, environment and disease to S Sengupta.

1. Alpert JS, Thygesen K, Antman E, Bassand JP. Myocardial infarction redefined-a consensus document of the joint European society of cardiology/American College of cardiology committee for the redefinition of myocardial infarction. J Am Coll Cardiol 2000; 36: 959-969.

2. Choudhary R, Mishra KP, Subramanyam C. Prevention of isoproterenol induced cardiac hypertrophy by eugenol. Indian J Clin Biochem 2006; 21: 107-113.

3. Chatterjee A, Mir SA, Dutta D, Mitra A, Pathak K, Sarkar S. Analysis of $p 53$ and NF- $\kappa B$ signaling in modulating the cardiomyocyte fate during hypertrophy. J Cell Physiol 2010; 226: 2543-2554.

4. Garciarena CD, Caldiz Cl, Portiansky EL, Chiappe de Cingolani GE, Ennis IL. Chronic NHE-1 blockade induces an antiapoptotic effect in the hypertrophied heart. $J$ Appl Physiol 2009; 106: 1325-1331.

5. Choudhury S, Bae S, Kumar SR, Ke Q, Yalamarti B, Choi JH et al. Role of AIF in cardiac apoptosis in hypertrophic cardiomyocytes from Dahl salt-sensitive rats. Cardiovasc Res 2010; 85: 28-37.

6. Sarkar S, Chawla-Sarkar M, Young D, Nishiyama K, Rayborn ME, Hollyfield JG et al. myocardial cell death and regeneration during progression of cardiac hypertrophy to heart failure. J Biol Chem 2004; 279: 52630-52642.

7. Griffiths ER, Friehs I, Scherr E, Poutias D, McGowan FX, del Nido PJ. Electron transport chain dysfunction in neonatal pressure-overload hypertrophy precedes cardiomyocyte apoptosis independent of oxidative stress. J Thorac Cardiovasc Surg 2010; 139: 1609-1617.

8. Xie MJ, Chang H, Wang YY, Zhang L, Song Z, Guo WG et al. Evidence that apoptotic signalling in hypertrophic cardiomyocytes is determined by mitochondrial pathways involving protein kinase C $\delta$. Clin Exp Pharmacol Physiol 2010; 37: 1120-1128.

9. Bloom S, Davis DL. Calcium as mediator of isoproterenol-induced myocardial necrosis. Am J Pathol 1972; 69: 459-470.

10. von Harsdorf R, Li PF, Dietz R. Signaling Pathways in Reactive Oxygen Species-Induced Cardiomyocyte apoptosis. Circulation 1999; 99: 2934-2941.

11. Zhang GM, Su SP, Wang Y, Li TD, Li XY, Tan $H$ et al. Effect of ischemic postconditioning on activation of p38 mitogen activated protein kinase and cardiocyte apoptosis in rats. Acta Acad Med Sin 2010; 32: 526-532.

12. Sun HJ, Li H, Zou Z, Shi XY. Protective effects of hydroxyethyl starch $130 / 0.4$ against myocardial ischemia/reperfusion injury in rats. Chin Med J 2011; 124: 291-297.

13. Morrison LE, Whittaker RJ, Klepper RE, Wawrousek EF, Glembotski CC. Roles for alphaB-crystallin and HSPB2 in protecting the myocardium from ischemia-reperfusioninduced damage in a KO mouse model. Am J Physiol Heart Circ Physiol 2004; 286: H847-H855.

14. Bullard B, Ferguson C, Minajeva A, Leake MC, Gautel M, Labeit D et al. Association of the chaperone alphaB-crystallin with titin in heart muscle. J Biol Chem 2004; 279: 7917-7924.

15. Maloyan A, Sanbe A, Osinska H, Westfall M, Robinson D, Imahashi K et al. Mitochondrial dysfunction and apoptosis underlie the pathogenic process in alpha-B-crystallin desminrelated cardiomyopathy. Circulation 2005; 112: 3451-3461.

16. Rajasekaran NS, Connell P, Christians ES, Yan LJ, Taylor RP, Orosz A et al. Human alpha B-crystallin mutation causes oxido-reductive stress and protein aggregation cardiomyopathy in mice. Cell 2007; 130: 427-439.

17. Jin JK, Whittaker R, Glassy MS, Barlow SB, Gottlieb RA, Glembotski CC. Localization of phosphorylated alphaB-crystallin to heart mitochondria during ischemia-reperfusion. $A m \mathrm{~J}$ Physiol Heart Circ Physiol 2008; 294: H337-H344.

18. Baldus S, Müllerleile K, Chumley P, Steven D, Rudolph V, Lund GK et al. Inhibition of xanthine oxidase improves myocardial contractility in patients with ischemic cardiomyopathy. Free Radic Biol Med 2006; 41: 1282-1288.

19. Kondo T, Ogawa Y, Sugiyama S, Ito T, Satake T, Ozawa T. Mechanism of isoproterenol induced myocardial damage. Cardiovasc Res 1987; 21: 248-254.

20. Lim DS, Roberts R, Marian AJ. Expression profiling of cardiac genes in human hypertrophic cardiomyopathy: insight into the pathogenesis of phenotypes. J Am Coll Cardiol 2001; 38: 1175-1180.

21. Shimizu S, Narita M, Tsujimoto Y. Bcl-2 family proteins regulate the release of apoptogenic cytochrome $c$ by the mitochondrial channel VDAC. Nature 1999; 399: 483-487.

22. Zheng $Y$, Shi $Y$, Tian $C$, Jiang $C$, Jin $H$, Chen $J$ et al. Essential role of the voltagedependent anion channel (VDAC) in mitochondrial permeability transition pore opening and cytochrome c release induced by arsenic trioxide. Oncogene 2004; 23: 1239-1247.

23. Glembotski CC. Endoplasmic reticulum stress in the heart. Circ Res 2007; 101: 975-984.

24. Okada K, Minamino T, Tsukamoto Y, Liao Y, Tsukamoto O, Takashima S et al. Prolonged endoplasmic reticulum stress in hypertrophic and failing heart after aortic constriction: possible contribution of endoplasmic reticulum stress to cardiac myocyte apoptosis. Circulation 2004; 110: 705-712. 
25. Sari FR, Watanabe K, Widyantoro B, Thandavarayan RA, Harima M, Zhang S et al. Partial inactivation of cardiac 14-3-3 protein in vivo elicits endoplasmic reticulum stress (ERS) and activates ERS-initiated apoptosis in ERS-induced mice. Cell Physiol Biochem 2010; 26: 167-178

26. Zhang C, Kawauchi J, Adachi MT, Hashimoto Y, Oshiro S, Aso T et al. Activation of JNK and transcriptional repressor ATF3/LRF1 through the IRE1/TRAF2 pathway is implicated in human vascular endothelial cell death by homocysteine. Biochem Biophys Res Commun 2001; 289: 718-724.

27. Pfahl M, Piedrafita FJ. Retinoid targets for apoptosis induction. Oncogene 2003; 22 9058-9062.

28. Bredesen DE, Rao RV, Mehlen P. Cell death in the nervous system. Nature 2006; 443 796-802.

29. Thuerauf DJ, Marcinko M, Gude N, Rubio M, Sussman MA, Glembotski CC. Activation of the unfolded protein response in infarcted mouse heart and hypoxic cultured cardiac myocytes. Circ Res 2006; 99: 275-282.

30. Lee YY, Hong SH, Lee YJ, Chung SS, Jung HS, Park SG et al. Tauroursodeoxycholate (TUDCA), chemical chaperone, enhances function of islets by reducing ER stress. Biochem Biophys Res Commun 2010; 397: 735-739.

31. Chis R, Sharma P, Bousette N, Miyake T, Wilson A, Backx PH et al. $\alpha$ Crystallin B prevents apoptosis following $\mathrm{H} 2 \mathrm{O} 2$ exposure in mouse neonatal cardiomyocytes. Am J Physio Heart Circ Physiol 2012; 303: H967-H978.

32. Ruan $Q$, Han S, Jiang WG, Boulton ME, Chen ZJ, Law BK et al. $\alpha B$-crystallin, an effector of unfolded protein response, confers anti-VEGF resistance to breast cancer via maintenance of intracrine VEGF in endothelial cells. Mol Cancer Res 2011; 9 : 1632-1643

33. Korkmaz S, Radovits T, Barnucz E, Hirschberg K, Neugebauer P, Loganathan S et al. Pharmacological activation of soluble guanylate cyclase protects the heart against ischemic injury. Circulation 2009; 120: 677-686.

34. Pfeffer MA, Pfeffer JM, Fishbein MC, Fletcher PJ, Spadaro J, Kloner RA et al. Myocardia infarct size and ventricular function in rats. Circ Res 1979; 44: 503-512.
35. Bohl S, Medway DJ, Schulz-Menger J, Schneider JE, Neubauer S, Lygate CA. Refined approach for quantification of in vivo ischemia-reperfusion injury in the mouse heart. $A m \mathrm{~J}$ Physiol Heart Circ Physiol 2009; 297: H2054-H2058.

36. Rivard AL, Steer CJ, Kren BT, Rodrigues CM, Castro RE, Bianco RW et al. Administration of tauroursodeoxycholic acid (TUDCA) reduces apoptosis following myocardial infarction in rat. Am J Chin Med 2007; 35: 279-295.

37. Pathak M, Sarkar S, Vellaichamy E, Sen S. Role of myocytes in myocardial collagen production. Hypertension 2001; 37: 833-840.

38. Shang L, Ananthakrishnan R, Li Q, Quadri N, Abdillahi M, Zhu Z et al. RAGE modulates hypoxia/reoxygenation injury in adult murine cardiomyocytes via JNK and GSK-3beta signaling pathways. PLoS One 2010; 5: e10092.

39. Bhowmick R, Halder UC, Chattopadhyay S, Chanda S, Nandi S, Bagchi P et al. Rotaviral enterotoxin nonstructural protein 4 targets mitochondria for activation of apoptosis during infection. J Biol Chem 2012; 287: 35004-35020.

40. Engel FB, Schebesta M, Duong MT, Lu G, Ren S, Madwed JB et al. p38 MAP kinase inhibition enables proliferation of adult mammalian cardiomyocytes. Genes Dev 2005; 19: 1175-1187.

41. Antrobus R, Borner GH. Improved elution conditions for native co-immunoprecipitation. PLoS One 2011; 6: e18218.

42. Saluja R, Saini R, Mitra K, Bajpai VK, Dikshit M. Ultrastructural immunogold localization of nitric oxide synthase isoforms in rat and human eosinophils. Cell Tissue Res 2010; 340: 381-388.

cc) (i) (-) $\Theta$ Cell Death and Disease is an open-access journal published by Nature Publishing Group. This work is licensed under a Creative Commons Attribution-NonCommercialNoDerivs 3.0 Unported License. To view a copy of this license, visit http://creativecommons.org/licenses/by-nc-nd/3.0/

Supplementary Information accompanies this paper on Cell Death and Disease website (http://www.nature.com/cddis) 